\title{
An evaluation of short-term outcomes after reoperations for anastomotic leakage in colon cancer patients
}

\author{
A. K. Warps ${ }^{1,2} \cdot$ J. W. T. Dekker ${ }^{3} \cdot$ P. J. Tanis ${ }^{4} \cdot$ R. A. E. M. Tollenaar ${ }^{1,2}$ (I)
}

Accepted: 13 July 2021 / Published online: 24 September 2021

(c) The Author(s) 2021

\begin{abstract}
Purpose Scarce data are available on differences among index colectomies for colon cancer regarding reoperation for anastomotic leakage (AL) and clinical consequences. Therefore, this nationwide observational study aimed to evaluate reoperations for AL after colon cancer surgery and short-term postoperative outcomes for the different index colectomies.

Methods Patients who underwent resection with anastomosis for a first primary colon carcinoma between 2013 and 2019 and were registered in the Dutch ColoRectal Audit were included. Primary outcomes were mortality, ICU admission, and stoma creation.

Results Among 39,565 patients, the overall AL rate was 4.8\% and ranged between 4.0\% (right hemicolectomy) and 15.4\% (subtotal colectomy). AL was predominantly managed with reoperation, ranging from $81.2 \%$ after transversectomy to $92.4 \%$ after sigmoid resection $(p<0.001)$. Median time to reoperation differed significantly between index colectomies (range 4-8 days, $p<0.001$ ), with longer and comparable intervals for non-surgical reinterventions (range 13-18 days, $p=0.747$ ). After reoperation, the highest mortality rates were observed for index transversectomy (15.4\%) and right hemicolectomy $(14.4 \%)$ and lowest for index sigmoid resection (5.6\%) and subtotal colectomy $(5.9 \%)(p<0.001)$. Reoperation with stoma construction was associated with a higher mortality risk than without stoma construction after index right hemicolectomy (17.7\% vs. $8.5 \%, p=0.001$ ). ICU admission rate was $62.6 \%$ overall (range 56.7-69.2\%), and stoma construction rate ranged between $65.5 \%$ (right hemicolectomy) and $93.0 \%$ (sigmoid resection).

Conclusion Significant differences in AL rate, reoperation rate, time to reoperation, postoperative mortality after reoperation, and stoma construction for AL were found among the different index colectomies for colon cancer, with relevance for patient counseling and perioperative management.
\end{abstract}

Keywords Anastomotic leakage $\cdot$ Reoperation $\cdot$ Colon cancer surgery $\cdot$ Mortality $\cdot$ Stoma

\section{Introduction}

Anastomotic leakage (AL) remains one of the most feared complications after colon cancer resection because of its consequences as sepsis and postoperative mortality [1-6].

R. A. E. M. Tollenaar

R.A.E.M.Tollenaar@lumc.nl

1 Department of Surgery, Leiden University Medical Center, Albinusdreef 2, 2333 ZA Leiden, The Netherlands

2 Dutch Institute for Clinical Auditing, Rijnsburgerweg 10, 2333 AA Leiden, The Netherlands

3 Department of Surgery, Reinier de Graaf Groep, Reinier de Graafweg 5, 2625 AD Delft, The Netherlands

4 Amsterdam UMC, Department of Surgery, University of Amsterdam, Cancer Centre Amsterdam, De Boelelaan 1117, 1081 HV Amsterdam, The Netherlands
$\mathrm{AL}$ is predominantly diagnosed within the first 2 weeks after surgery [7-11]. Patients suffering from AL after colorectal surgery often require a reintervention, which commonly includes a reoperation with stoma creation [3-7].

Although the AL rate is lower after colon cancer resection compared to rectal cancer surgery $[12,13]$, it has been suggested that $\mathrm{AL}$ after colon resection presents earlier, with more severe complications and a higher mortality rate compared to AL after rectal cancer surgery [2]. This is likely related to the intraperitoneal location of the anastomosis after colon cancer resection with a high risk of generalized peritonitis, while leakages after (low) anterior resection often result in contained extraperitoneal abscess formation $[2,14]$. In addition, the estimated AL rates, reoperation rates, and postoperative outcomes vary among the different sites of anastomosis [2, 4-7, 12-17]. 
Limited data is available on differences in management and clinical consequences of AL for different types of colon cancer resection. Therefore, the present study aimed to provide insights into the type and timing of reoperation for $\mathrm{AL}$ in colon cancer patients who underwent different types of colectomy with primary anastomosis and subsequent postoperative outcomes.

\section{Methods}

This nationwide population-based cohort study was performed with data from the Dutch Colorectal Audit (DCRA). The DCRA is a nationwide clinical audit that registers all patients who underwent surgery for primary colorectal cancer in the Netherlands. The registry is known for its high data completeness (nationwide coverage $>95 \%$ ) and external data verification to assure high validity [18]. Ethical approval and informed consent were not required, as stated by Dutch law.

\section{Study population}

All patients who underwent elective surgery with primary anastomosis creation for a first primary colon carcinoma between January 1, 2013 and December 31, 2019 and registered in the DCRA were potentially eligible.

Data extracted from the DCRA comprised characteristics concerning patient, tumor, surgical, and follow-up information. The 30-day follow-up was registered until December 31, 2017, and from January 1, 2018, the 90-day follow-up was registered.

\section{Outcomes and definitions}

Index surgical procedures were divided into right hemicolectomy, transversectomy, left hemicolectomy, sigmoid resection, and subtotal colectomy. AL was defined as a defect of the intestinal wall or abscess at the site of the colorectal anastomosis, for which a reintervention was required within 30 to 90 days from primary resection. Since the date of AL diagnosis is not available in the DCRA, the present study reports the follow-up from index colectomy to reintervention. Reinterventions were divided into two categories: (1) surgical reinterventions including laparoscopic and open surgical reinterventions and (2) non-surgical reinterventions including radiologic, endoscopic, and other unspecified reinterventions.

For each type of index colectomy, the occurrence of AL, type of reintervention, and timing of reintervention were determined. Primary outcomes after reoperation were mortality, ICU admission, and stoma construction. Secondary outcomes were prolonged hospital stay (a primary hospital stay of more than 14 days (LOS $>14$ days)), readmission, stoma creation per type (defunctioning ileo- or colostomy, end ileo- or colostomy), and mortality for patients with and without stoma creation during reoperation.

\section{Statistical analyses}

Baseline study population characteristics are reported for patients with and without AL. Outcomes after reoperation were reported for the total study population and for each type of index colectomy. Sub-analyses were performed to assess differences in outcomes for reoperation performed during the weekend vs. week and for different annual hospital volumes. Since the Dutch standard states that hospitals should perform at least 50 colonic resections per year [19], volumes were categorized into low- $(<50)$, low-intermediate (50-75), intermediate-high (76-100), and high- ( $>100)$ volume hospitals. Categorical and dichotomous variables are reported as absolute numbers with percentages and were compared using the Pearson chi-square test or Fisher's exact test. Continuous variables are reported as median with interquartile range (IQR), and a Kruskal Wallis rank-sum test was used to assess statistical significance.

The time interval between surgery and reoperation was calculated using the date of surgery and the date of reintervention. To visualize the timing of reoperation, the number of reoperations per 2 days was plotted for each type of resection separately. Statistical significance was defined as a $p$-value $<0.05$. RStudio version 1.4.1106 (2021) was used for statistical analyses.

\section{Results}

\section{Study population}

All patients who underwent surgery for a first primary colon carcinoma between January 1, 2013 and December 31, 2019 and registered in the DCRA were potentially eligible for this study $(n=52,035)$ (Fig. 1). For the purpose of this study, the following patients were excluded: patients with a synchronous colorectal carcinoma $(n=1770)$; patients who underwent emergency surgery $(n=6806)$, who underwent a local excision $(n=39)$; patients in whom no primary anastomosis was constructed $(n=2659)$; patients with a prior stoma of any type as bridge to surgery which was not reversed during the elective colectomy or patients with a stoma of any type constructed during elective colectomy $(n=1178)$; patients with missing data on $\operatorname{AL}(n=3)$ and patients who underwent a proctocolectomy $(n=15)$ were excluded. After exclusion, a total of 39,565 patients were included in the study.

The overall AL rate was $4.8 \%$ and baseline characteristics are displayed in Table 1. Compared to patients without $\mathrm{AL}$, those with $\mathrm{AL}$ were more frequently male $(62.5 \%$ 
Fig. 1 Study flowchart. Synchronous CRC synchronous colorectal cancer, AL anastomotic leakage

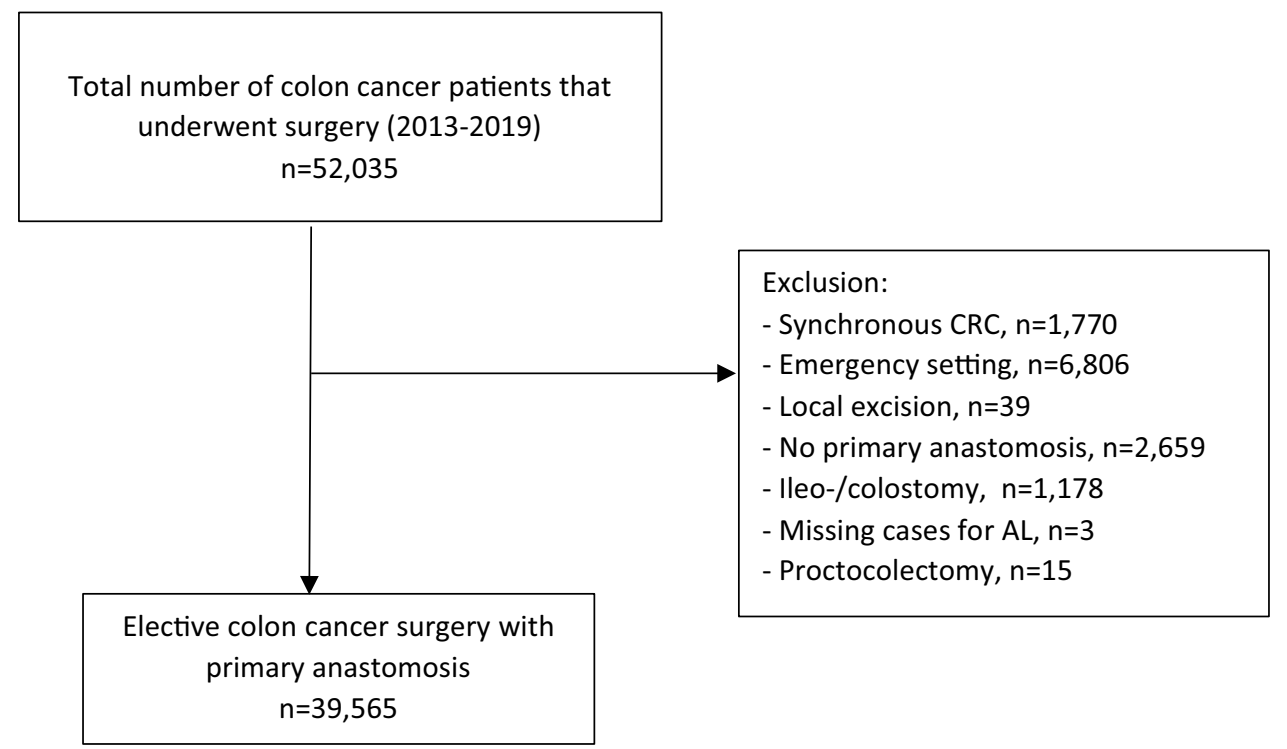

vs. $52.2 \%, p<0.001)$, obese (BMI $\geq 30 \mathrm{~kg} / \mathrm{m}^{2} 22.7 \%$ vs. $19.9 \%, p=0.009$ ), less healthy (ASA III $+31.7 \%$ vs. $24.8 \%$, $p<0.001$; CCI II $+32.7 \%$ vs. $28.0 \%, p<0.001)$, more often presented with tumor-related complications such as anemia or peritumoral abscess $(32.7 \%$ vs. $27.4 \%, p=0.006)$ and had a more advanced tumor stage (T4 $13.5 \%$ vs. $10.0 \%, p<0.001$ and M1 $11.0 \%$ vs. $7.4 \%, p<0.001)$. Regarding treatment characteristics, these patients more often received neoadjuvant chemotherapy $(3.1 \%$ vs. $1.6 \%, p<0.001)$, more often underwent an open resection $(23.8 \%$ vs. $16.5 \%, p<0.001)$, a multivisceral resection $(11.5 \%$ vs. $6.5 \%, p<0.001)$ or an additional resection for metastasis (5.5\% vs. $2.8 \%$, $p<0.001)$.

The total number of patients treated at low-volume hospitals was 5564, which was 7454 for low-intermediate-volume hospitals, 8163 for intermediate-high volume hospitals, and 18,384 for high-volume hospitals. The highest AL rate was found for low volume hospitals $(6.0 \%)$, which was significantly higher if compared to the other volume categories (lowintermediate volume hospitals $4.3 \%$, intermediate-high volume hospitals $4.7 \%$, high-volume hospitals $4.7 \%, p<0.001$ ).

\section{Anastomotic leakage and reintervention}

The AL rate differed significantly among the different index procedures. The lowest AL rate was found for patients who underwent a right hemicolectomy (4.0\%), followed by sigmoid resection $(4.9 \%)$, left hemicolectomy $(6.7 \%)$, transversectomy (7.4\%), and a subtotal colectomy $(15.4 \%)$ ( $p<0.001$ ) (Fig. 2A). Reinterventions were predominantly surgical, ranging from $81.2 \%$ for transversectomy to $92.4 \%$ for sigmoid resection $(p<0.001)$ (Fig. 2B). The median time to reoperation differed significantly among the index colectomies with the shortest time-interval to reoperation for sigmoid resection (4 days, IQR 3-6), followed by hemicolectomy (both left and right: 6 days, IQR 4-9) and transversectomy (6 days, IQR 3-9), and the longest for subtotal colectomy ( 8 days, IQR $4-11)(p<0.001)$. Figure 3 demonstrates that most reoperations were performed on postoperative days 3-4 ( $n=156$ for right hemicolectomy; $n=65$ for left hemicolectomy, $n=240$ for sigmoid resection, and $n=10$ for subtotal colectomy).

Non-surgical reinterventions for managing AL were most frequently performed for patients who initially underwent transversectomy $(18.8 \%)$ or right hemicolectomy $(17.1 \%)$. The median time to non-surgical reinterventions did not differ between the index surgical procedures: 13 days (IQR 9-18) for right hemicolectomy, 13.5 days (IQR 10-17) for sigmoid resection, 16 days (IQR 10-20) for left hemicolectomy, 16 days (IQR 14-26) for subtotal colectomy, and 18 days (IQR 10-26) for transversectomy $(p=0.784)$.

\section{Short-term outcomes after reoperations for anastomotic leakage}

Figure 4A shows the short term-outcomes after reoperation for AL $(n=1592)$, stratified for index colonic resection type as well as for the overall group.

During reoperation, a stoma was frequently constructed $(79.5 \%)$. This varied significantly across the different procedures ( $p<0.001)(65.5 \%$ (right hemicolectomy) vs. $75.0 \%$ (transversectomy) vs. 80.4\% (subtotal colectomy) vs. $85.7 \%$ (left hemicolectomy) vs. 93.0\% (sigmoid resection)). Of all reoperations performed, 320 patients underwent a reoperation without stoma construction $(70.0 \%$ of these patients initially underwent a right hemicolectomy), 437 received a defunctioning stoma (42.6\% of these patients initially 
Table 1 Baseline study population characteristics of patients who underwent colon cancer resection, stratified for anastomotic leakage (nonanastomotic leakage (non-AL) versus anastomotic leakage (AL))

\begin{tabular}{|c|c|c|c|c|}
\hline & & Total study populati & $(n=39,565)$ & \\
\hline & & No-AL $(n=37,658)$ & AL $(n=1907)$ & $p$-value \\
\hline Age & $\geq 75$ & $13,526(35.9)$ & $698(36.6)$ & 0.565 \\
\hline & Missing & 6 & 0 & \\
\hline Sex & Male & $19,642(52.2)$ & $1,191(62.5)$ & $<0.001$ \\
\hline & Missing & 11 & 0 & \\
\hline BMI & $<18.5$ & $1,103(2.9)$ & $51(2.7)$ & 0.009 \\
\hline & $18.5-30.0$ & $29,054(77.2)$ & $1,421(74.5)$ & \\
\hline & $\geq 30$ & $7,484(19.9)$ & $433(22.7)$ & \\
\hline & Missing & 17 & 2 & \\
\hline ASA & $\mathrm{III}+$ & $9,348(24.8)$ & $604(31.7)$ & $<0.001$ \\
\hline & Missing & 9 & 0 & \\
\hline CCI & $\mathrm{II}+$ & $10,619(28.0)$ & $626(32.7)$ & $<0.001$ \\
\hline Tumor complication ${ }^{\mathrm{a}}$ & & $10,316(27.4)$ & $579(32.7)$ & 0.006 \\
\hline & Missing & 64 & 1 & \\
\hline Neoadjuvant chemotherapy & & $610(1.6)$ & $60(3.1)$ & $<0.001$ \\
\hline & Missing & 848 & 45 & \\
\hline Approach & Open & $6,209(16.5)$ & $456(23.8)$ & $<0.001$ \\
\hline & Laparoscopic $^{\text {b }}$ & $31,008(82.3)$ & $1432(75.1)$ & \\
\hline & Missing & 441 & 23 & \\
\hline Type of resection & & & & \\
\hline Right hemicolectomy & & $19,507(51.8)$ & $823(43.2)$ & $<0.001$ \\
\hline Transversectomy & & $813(2.2)$ & $65(3.4)$ & \\
\hline Left hemicolectomy & & $4,106(10.9)$ & $295(15.5)$ & \\
\hline Subtotal colectomy & & $318(0.8)$ & $58(3.0)$ & \\
\hline Sigmoid resection & & $12,729(33.8)$ & $650(34.1)$ & \\
\hline & Missing & 185 & 16 & \\
\hline Stoma as bridge to surgery & No, stoma created as bridge to surgery & $37,325(99.1)$ & $1,884(98.8)$ & 0.184 \\
\hline & Yes, reversed during elective colectomy & $333(0.9)$ & $23(1.2)$ & \\
\hline Multivisceral resection & & $2,461(6.5)$ & $219(11.5)$ & $<0.001$ \\
\hline & Missing & 15 & 0 & \\
\hline Additional resection for metastases & & $1,041(2.8)$ & $105(5.5)$ & $<0.001$ \\
\hline & Missing & 28 & 1 & \\
\hline T-stage & $\mathrm{T} 1-2$ & $13,980(37.1)$ & $599(31.4)$ & $<0.001$ \\
\hline & T3 & $19,726(52.4)$ & $1046(54.9)$ & \\
\hline & $\mathrm{T} 4$ & $3,766(10.0)$ & $257(13.5)$ & \\
\hline & Missing & 186 & 5 & \\
\hline N-stage & No & $25,307(67.2)$ & $1261(66.1)$ & 0.326 \\
\hline & $\mathrm{N} 1-2$ & $12,235(32.5)$ & 641 (33.6) & \\
\hline & Missing & 116 & 5 & \\
\hline M-stage & M- & $34,859(92.6)$ & $1698(89.0)$ & $<0.001$ \\
\hline & M1 & $2,799(7.4)$ & $209(11.0)$ & \\
\hline
\end{tabular}

${ }^{\text {a }}$ Tumor complications are preoperative complications caused by the tumor, including peri-tumoral abscess, anemia, perforation, obstruction/ileus ${ }^{b}$ Laparoscopic procedures include conventional and robot-assisted laparoscopic procedures. Missing values less than $10 \%$ are only presented as absolute numbers. A Pearson chi-square test was used to calculate the $p$-value

underwent a sigmoid resection), and 819 received an end stoma ( $42.4 \%$ of these patients initially underwent a sigmoid resection). The type of stoma constructed during reoperation is demonstrated in Fig. 4B. In right hemicolectomy patients, an end stoma was most often constructed during reoperation $(41.3 \%)$, although a substantial proportion of the patients received no stoma during reoperation $(34.3 \%)$. The highest proportions of end stoma construction during reoperation 


\section{a $18 \%$}

$16 \%$

\section{$14 \%$}

$12 \%$

$10 \%$

$8 \%$

$6 \%$

$4 \%$

$2 \%$

$0 \%$

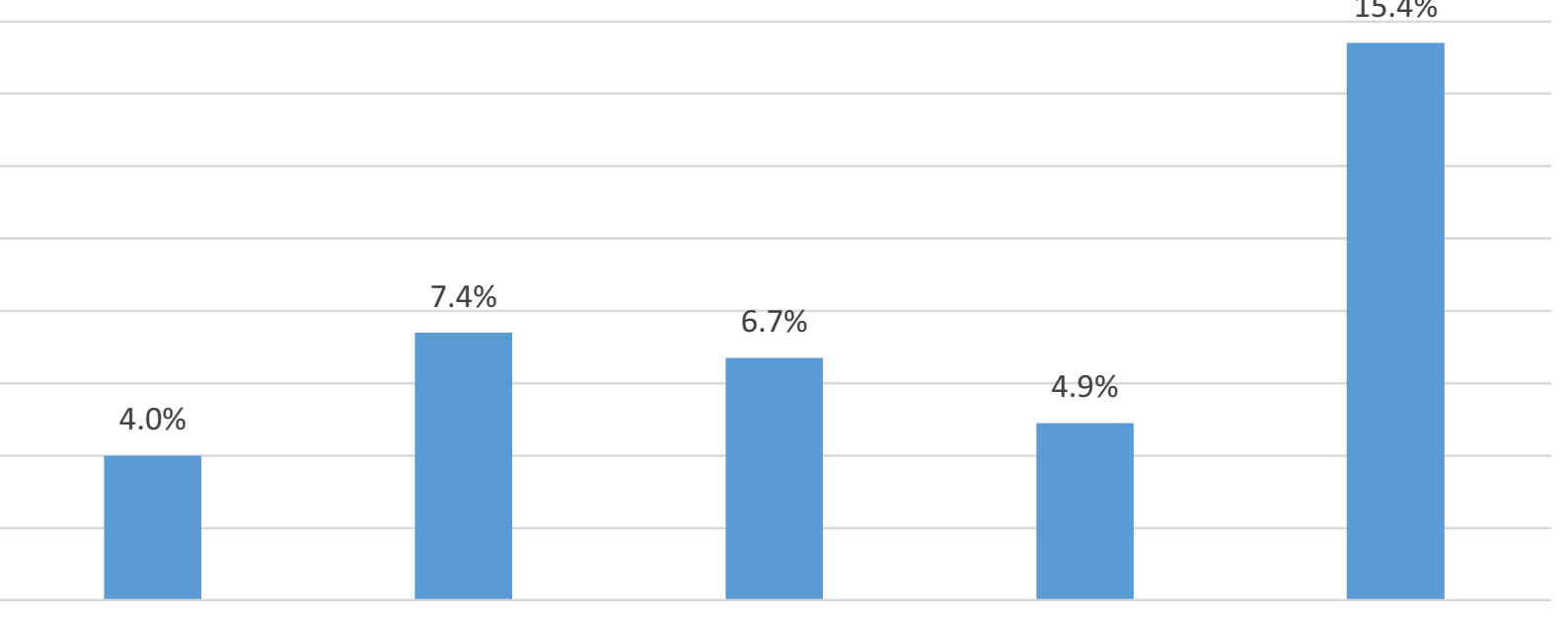

Right hemicolectomy Transversectomy Left hemicolectomy Sigmoid resection Subtotal colectomy

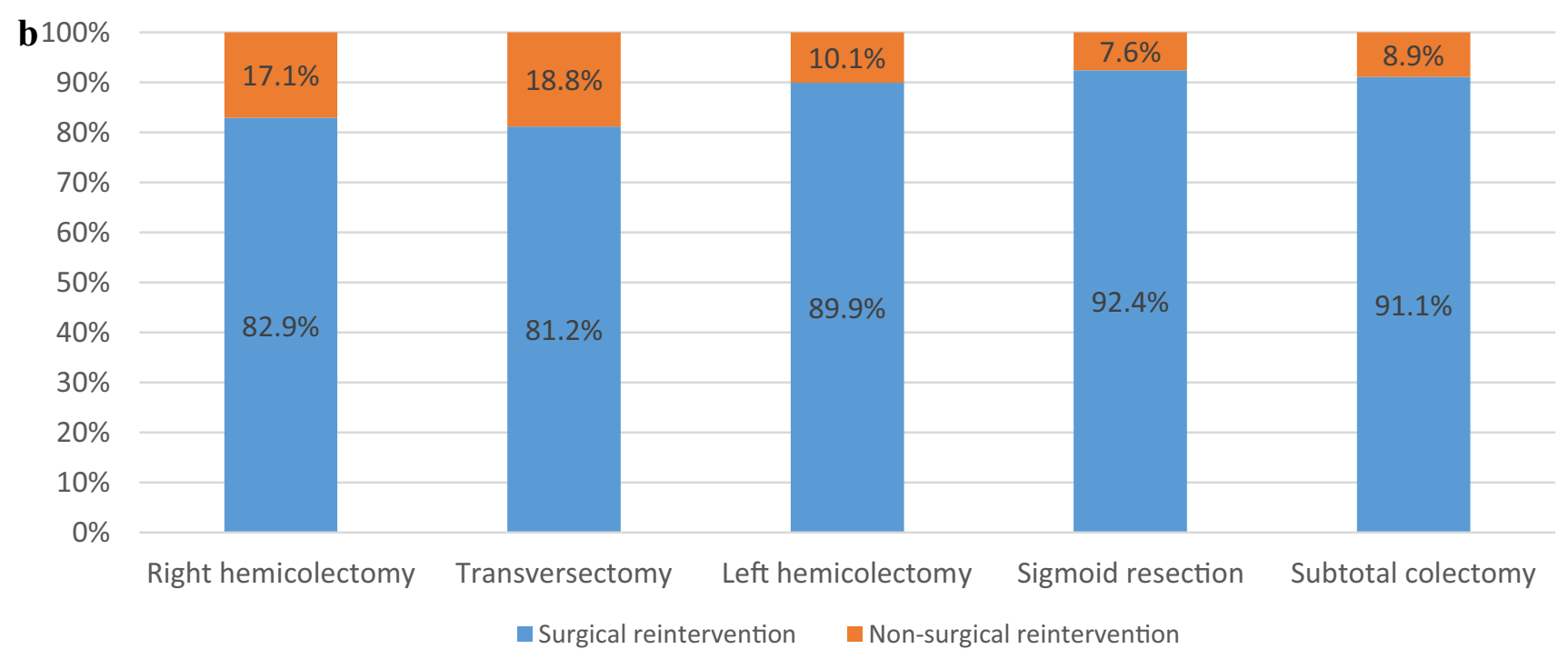

Fig. 2 Title Figure 2A, Anastomotic leakage for each type of index surgical procedure

Legend of Figure 2A, The anastomotic leakage rate after each type of index colon cancer resection. Patients with a unknown type of index colectomy $(\mathrm{N}=201)$ were excluded from analyses, resulting in the inclusion of 39,565 patients (right hemicolectomy $\mathrm{N}=20,330$, transversectomy $\mathrm{N}=878$, left hemicolectomy $\mathrm{N}=4,401$, sigmoid resection $\mathrm{N}=13,379$ and subtotal colectomy $\mathrm{N}=376$ )

Title Figure 2B, Type of reintervention for each type of index surgical procedure

were observed for index left hemicolectomy (62.6\%) and sigmoid resection $(60.7 \%)$.

Overall, patients who received a stoma during reoperation had a similar mortality rate compared to patients who did not receive a stoma $(7.8 \%$ vs. $11.1 \%, p=0.104)$. After reoperation, overall mortality and ICU admission rates were $10.5 \%$ and $62.6 \%$, respectively, and varied significantly
Legend Figure 2B, Presents for each index procedure the type of reintervention for patients who suffered from anastomotic leakage. The type of reintervention was subdivided into non-surgical reinterventions (e.g. image guided, endoscopic or other reinterventions) and surgical reinterventions. Patients with a unknown type of index colectomy $(\mathrm{N}=16)$ or unknown type of reintervention $(\mathrm{N}=71)$ were excluded from analyses, resulting in the inclusion of 1,820 patients (right hemicolectomy $\mathrm{N}=791$, transversectomy $\mathrm{N}=64$, left hemicolectomy $\mathrm{N}=287$, sigmoid resection $\mathrm{N}=622$, subtotal colectomy $\mathrm{N}=56$ )

across the index procedures $(p=0.001$ and $p<0.001)$. The highest mortality and ICU admission rates were found in patients who initially underwent a transversectomy $(15.4 \%$ and $69.2 \%$ ) or right hemicolectomy (14.5\% and $66.3 \%$ ), followed by patients who underwent a left hemicolectomy $(11.2 \%$ and $64.7 \%)$, subtotal colectomy $(5.9 \%$ and $62.7 \%$ ), and sigmoid resection (5.6\% and $56.7 \%$ ). Figure 5 


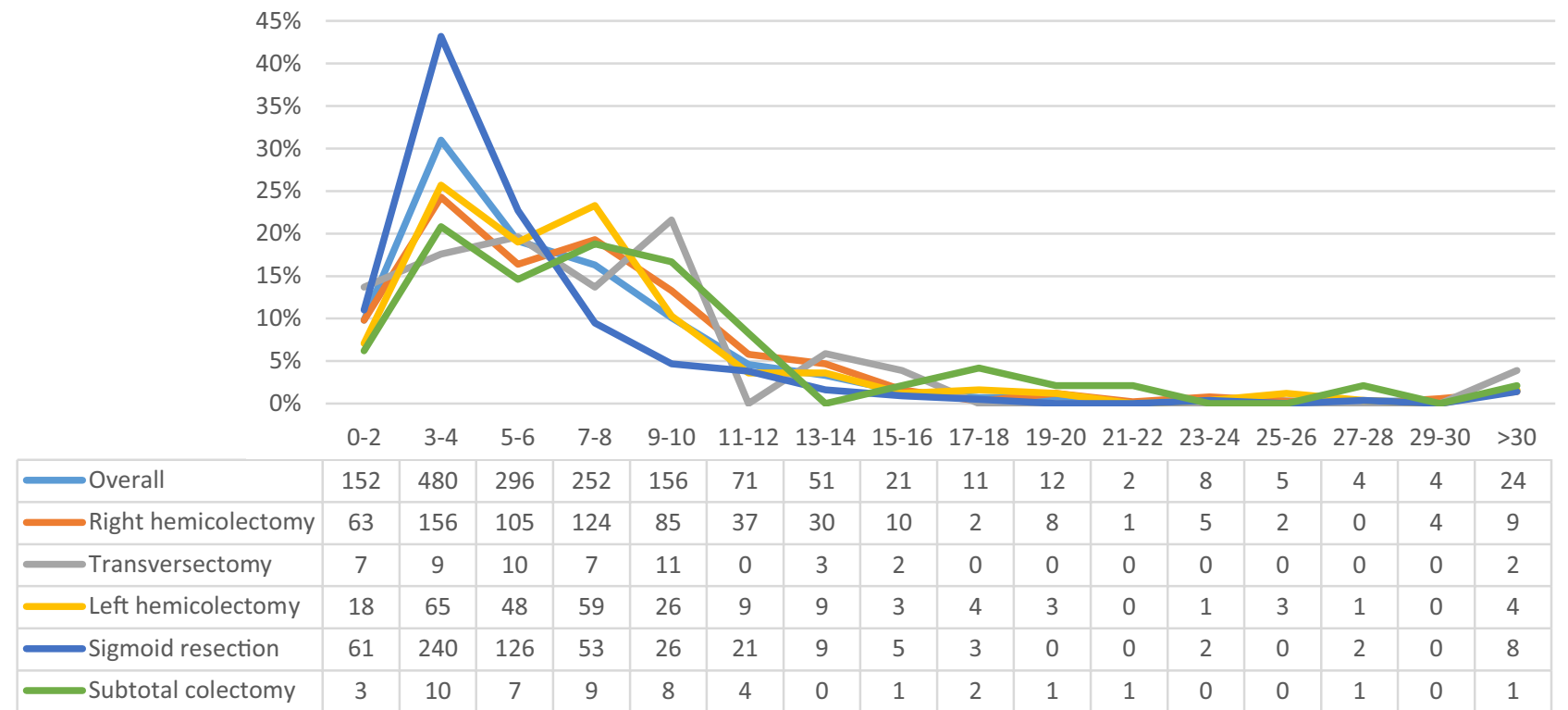

Fig. 3 Title Figure 3A, Number of reoperations per time-interval from index surgical procedure to reoperation

Legend Figure 3, Number and percentages of reoperations performed per time interval ( 2 days) between index surgical procedure and reoperation. Patients with an unknown type of index colectomy $(\mathrm{N}=16)$ or

unknown date of reoperation $(\mathrm{N}=43)$ were excluded. Overall number of patients included $\mathrm{N}=1,549$ and for right hemicolectomy $\mathrm{N}=641$, transversectomy $\mathrm{N}=51$, left hemicolectomy $\mathrm{N}=253$, sigmoid resection $\mathrm{N}=556$, and subtotal colectomy $\mathrm{N}=48$

demonstrates the mortality rate for each index procedure, in which patients were stratified for stoma construction during reoperation. For index right hemicolectomy, patients receiving a stoma during reoperation for AL had a significantly higher mortality rate than patients who did not receive a stoma $(17.7 \%$ vs. $8.5 \%, p=0.001)$.

A primary hospital stay of more than 14 days in patients that underwent reoperation for AL during the same admission occurred in $59.4 \%$. For the different index colectomies, this percentage varied between $54.1 \%$ (sigmoid resection) and $70.6 \%$ (subtotal colectomy) ( $p=0.004$ ). Of the patients who underwent a reoperation for AL during primary admission, $13.7 \%$ was readmitted within 30 days, which was comparable among the different index colectomies $(p=0.156)$.

Patients reoperated during the weekend $(n=410)$ had comparable outcomes to patients $(n=1139)$ reoperated on weekdays (stoma construction ( $p=0.387$ ), ICU admission $(p=0.752)$, mortality $(p=0.525)$, LOS $>14(p=0.181)$ and readmission $(p=0.663)$ ). Analyzing the influence of hospital volume on adverse events after reoperation $(n=1608)$ neither revealed significant differences regarding stoma construction $(p=0.482)$, ICU admission $(p=0.673)$, mortality $(p=0.860)$, LOS $>14$ days $(p=0.072)$, and readmission $(p=0.667)$.

\section{Discussion}

In the present population-based study, we provide insights into the daily practice and postoperative clinical course of patients that suffered from AL after colonic resection for

primary colon cancer. We found an $\mathrm{AL}$ rate of $4.8 \%$, ranging from $4.0 \%$ for right hemicolectomy to $15.4 \%$ for subtotal colectomy. AL was mostly managed with surgical reintervention (84.3\%) and stoma construction (79.5\%), but significant differences in reoperation and stoma rates were found for the different index colectomies. Also, the median time to reoperation differed significantly. Mortality rates of about $15 \%$ after reoperation were observed for index transversectomy and right hemicolectomy, and this was $6 \%$ for index sigmoid resection and subtotal colectomy. In addition, it was found that reoperations for AL after colectomy are accompanied by a substantial ICU admission rate and prolonged stay in hospital rate, which varies significantly among the different surgical procedures. Transversectomy patients demonstrated the most severe complicated course (e.g., higher ICU admission rate and mortality rate) but also are more often treated non-surgically compared to other colectomies. Right hemicolectomy patients suffering from AL were less commonly managed with reoperation and stoma creation than other colectomy types but showed a substantial mortality rate. After reoperation, the mortality rate was significantly higher for these patients when they received a stoma during reoperation, which might be related to the abdominal contamination.

Compared to rectal cancer resection, construction of an anastomosis in colonic resection might be technically less demanding, and $\mathrm{AL}$ rates are generally lower. However, when AL occurs after segmental colectomy, it can easily spread throughout the peritoneal cavity, causing generalized 


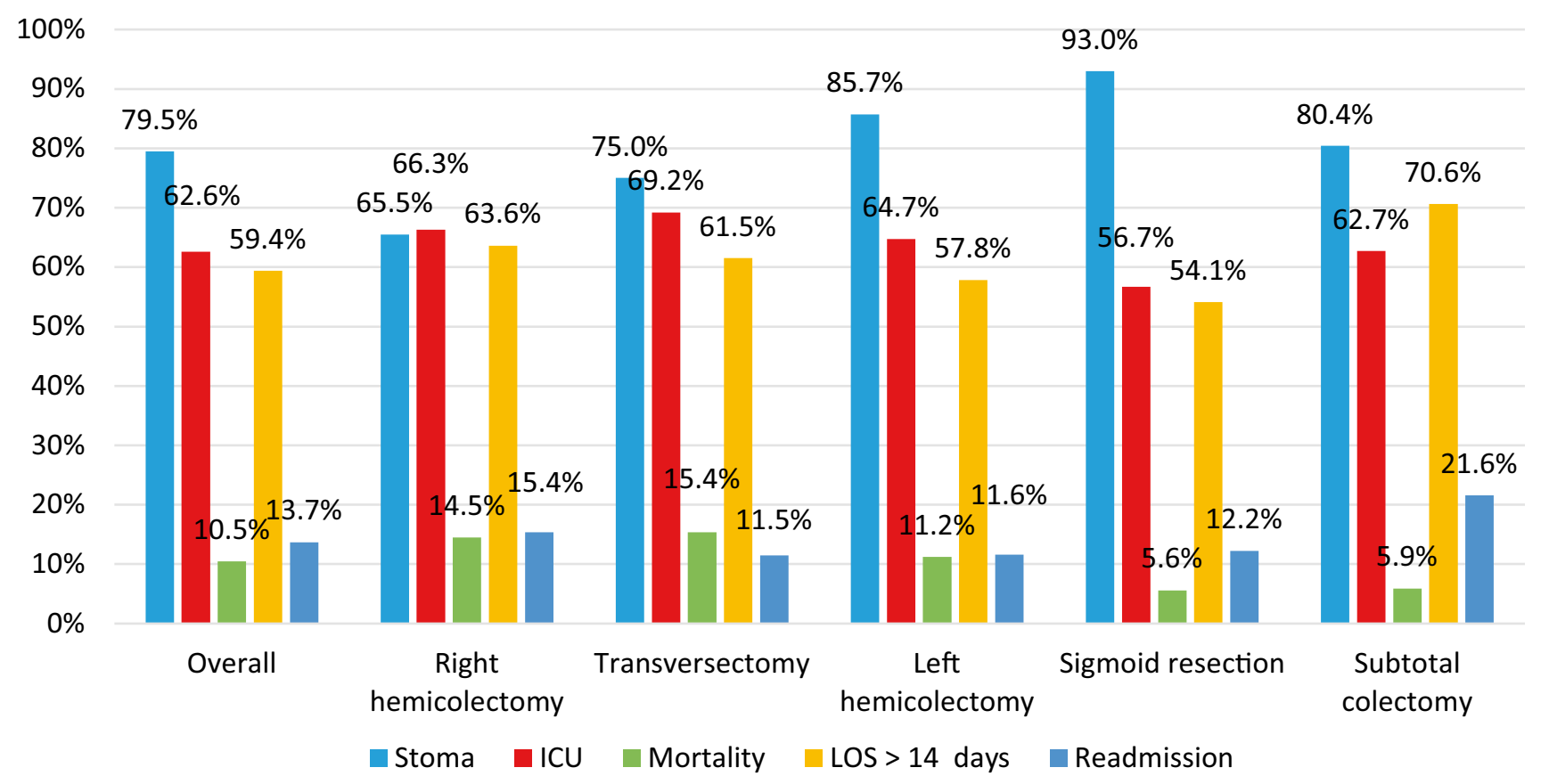

Fig. 4 Title of Figure 4A, Short-term outcomes after reoperation for each type of index surgical procedure

Legend of figure 4A, Patients with an unknown type of colectomy were excluded $(n=16)$ from analyses, resulting in the inclusion of 1592patients. Title figure 4B, Type of stoma creation during reoperation for each type of index surgical procedure

Legend of Figure 4B, Demonstrates the stoma creation rate during

peritonitis and rapidly developing sepsis [2, 14]. This might be the reason for the overall high mortality rates following reoperation, as observed in the present study. Interestingly, these mortality rates were more than twice as high after initial rightsided resections compared to sigmoid resection and subtotal colectomy. Bakker et al. and Veyrie et al. found a decreased risk of AL after a right hemicolectomy, but with contrasting results regarding the AL-related mortality [2, 17]. Similar to our study, it was demonstrated that a subtotal colectomy has the highest risk of AL compared to all other types of colectomy, up to $23 \%[2,7,20]$. Surprisingly, mortality after reoperation for AL following index subtotal colectomy was low in the present study. This is difficult to explain, but a potential explanation for this finding might be the type of anastomosis, differences in intestinal microbiome [13, 21, 22], and a more contained bacterial contamination in the pelvic cavity.

A recent snapshot study found an $\mathrm{AL}$ rate of $7.4 \%$ after right hemicolectomy, while we found an $\mathrm{AL}$ rate of $4.0 \%$ [23]. This might be due to difference in definition of $\mathrm{AL}$ and completeness of registration. In line with previous studies, we found similar patient and tumor-related risk factors, besides surgical factors such as multivisceral resection and simultaneous metastasectomy [4-6, 8, 23-25]. In contrast to the FOXTROT trial [26], we found that neoadjuvant reoperation for anastomotic leakage for each type of index procedure. Defunctioning stoma includes both a defunctioning ileo- and colostomy, and end stoma includes both an end ileo- and colostomy. Patients were excluded if the type of colectomy was unknown $(\mathrm{N}=16)$ or if information on stoma creation during reoperation $(\mathrm{N}=4)$ was missing, resulting in the inclusion of 1,586 patients

chemotherapy was significantly associated with AL. This was also found by Midura et al. in multivariable analysis [6].

Previous studies demonstrated that early AL (before day 6) is associated with more life-threatening complications and mortality than late AL $[8,9]$. We found that most reoperations were performed on days $3-4$. Our results are in line with the current literature that reports a median time to reintervention for AL ranging from 4.0 to 12.7 days [7-11]. Delayed diagnosis of AL with a difference in postoperative care might explain varying time intervals. In addition, our results suggest that this might also be related to the type of primary surgical procedure as a potential consequence of technical aspects of the anastomosis (e.g., configuration, type of stapling, location) and differences in for example vascularization. Sparreboom et al. found that surgical difficulties during anastomosis construction resulted in more early AL, whereas poor patient and tissue condition was associated with late leakage [8].

It has been demonstrated that AL after colon cancer surgery is a major complication leading to significant sepsis. With the intent to prevent further deterioration in the postoperative course and ultimately postoperative death, a stoma is constructed during reoperation $[3,4,11,27]$. This was confirmed by our study. We found a comparable overall 


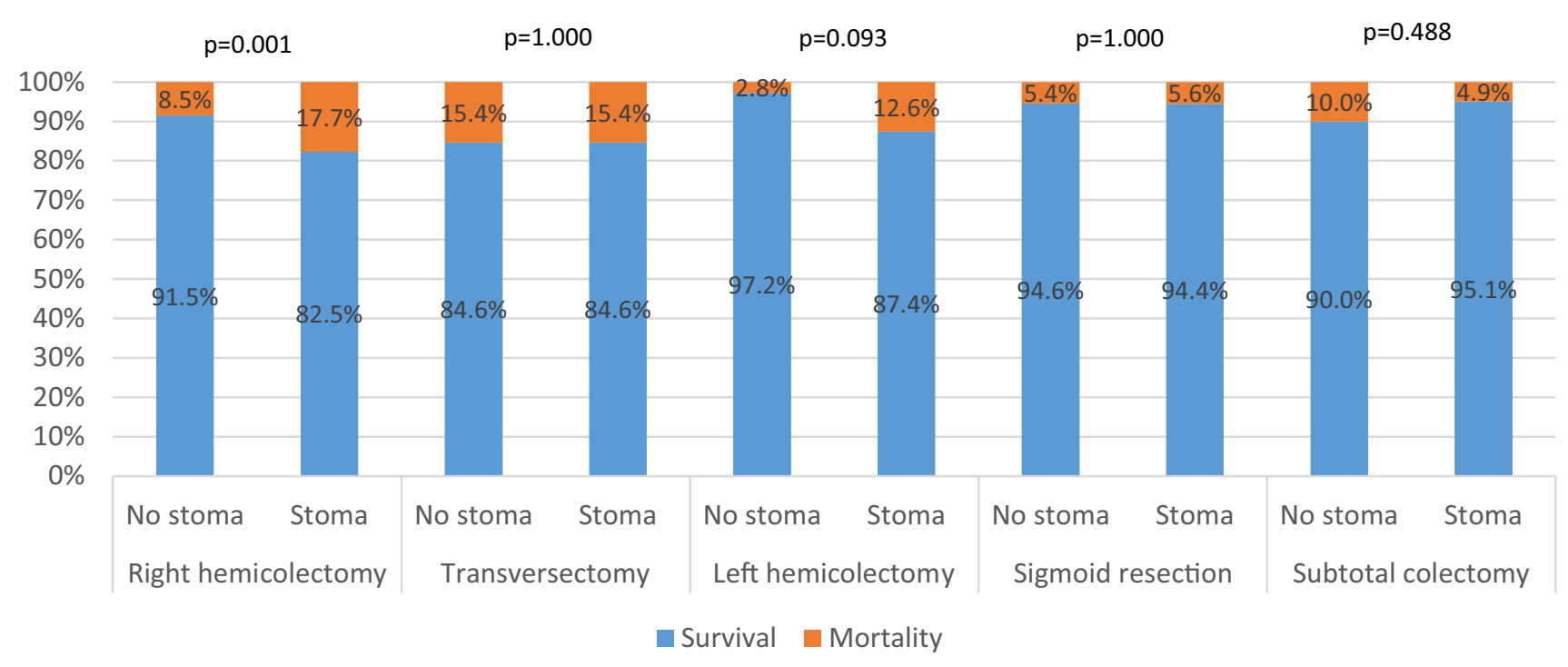

Fig. 5 Title of Figure 5, Mortality rate after reoperation with stoma creation versus without stoma creation

Legend of Figure 5, Patients with unknown data regarding colectomy

type $(n=16)$ or stoma construction during reoperation $(n=4)$ were excluded, resulting in the inclusion of 1586 patients. Statistical significance was calculated with a Fisher exact test

stoma rate to previous studies after AL of $60-80 \%[4,10$, $11,27]$. Our results demonstrated that the stoma rate ranged from 55.5 to $90.6 \%$ among the types of index surgical procedures, with various proportions of defunctioning and end stomas. This might be explained by the anatomic location of the anastomosis after resection. Krarup et al. found that less than a third of the colon cancer patients who suffered from AL underwent anastomotic repair, and that $14.6 \%$ of the anastomoses could be salvaged. They found no significant differences in 30-day mortality and long-term mortality in multivariable analyses for anastomosis takedown and salvage [27].

Although our study did not include patients who received a stoma during the index surgical procedure, we demonstrated no significant differences in mortality for patients with and without defunctioning stoma creation during reoperation except for right hemicolectomy. A defunctioning stoma is not routinely constructed for AL after a right hemicolectomy. A redo of the anastomosis is frequently performed, and patients in worse clinical condition generally receive a stoma. This causes a patient selection for stoma construction during reoperation for AL after a right hemicolectomy. The comparable mortality rates for patients with and without stoma creation during reoperation show that patient selection is adequate. During reoperation, it should be taken into account that stoma itself also lead to a significant stoma complication rate [28, 29] and decrease in quality of life [29, 30], which stresses the importance of not routinely constructing a stoma during reoperations for $\mathrm{AL}$ after colectomy, especially in case of right hemicolectomy.

Several limitations need to be addressed. The Dutch ColoRectal Audit registers only short-term outcomes (e.g., 90 days). For this reason, we could not evaluate the longterm outcomes after AL, such as AL-rate after 90 days, overall survival, disease-free survival, and stoma reversal. In addition, no information is registered regarding the date of complication, clinical parameters, additional radiologic imaging for identifying AL, or multiple reoperations. Therefore, we could only evaluate the time interval between surgery and reintervention, and a delay in diagnosis and treatment could not be assessed. Other lacking information is related to the cause of $\mathrm{AL}$, the type and technique of anastomosis constructed, the severity of illness during reoperation, and reoperation during daytime or nighttime, since audits are limited in the number of variables related to registration burden. Therefore, datasets need to be concise with a focus on most relevant variables required for the aim of clinical auditing and improvement of quality of care [31]. Furthermore, due to selection bias (confounding by indication) between the types of primary colon resection, it is impossible to attribute differences in outcome solely to the resection type. Instead, we can only reflect on daily practice and postoperative clinical course. The main strength of this study is the robustness of the data enabling a detailed insight into short-term outcomes after AL and reinterventions concerning primary colon resection. 


\section{Conclusion}

The present study provides insights into the daily practice of managing $\mathrm{AL}$ after colon cancer resection as well as the postoperative clinical course after reoperations. The occurrence of AL, the type of reintervention, and the outcomes after reoperations vary among the different colectomy types performed. These findings highlight the importance of assessing diagnosis, treatment, and outcomes of AL for the different types of index colectomies in future studies to optimize outcomes of surgical care. Besides, selective stoma construction during reoperation for $\mathrm{AL}$ is currently applied in a safe way in the Netherlands, since a comparable mortality rate was observed for patients who did and who did not receive a stoma during reoperation.

Acknowledgements The authors would like to thank all the surgeons, data managers, physician assistants, and administrative nurses who have registered all patients in the DCRA.

Author contribution Study concepts and design: Warps, Dekker, Tanis, and Tollenaar. Data acquisition: Warps. Statistical analysis: Warps. Data interpretation: Warps, Dekker, Tanis, and Tollenaar. Drafting the manuscript: Warps. Supervision of manuscript: Tollenaar. Critical review and feedback on the manuscript: Dekker, Tanis, and Tollenaar.

Availability of data and material The data supporting the results of the present study are only available from the authors upon reasonable request and with permission of the Dutch Institute for Clinical Auditing and the Dutch ColoRectal Audit Board. Data is not publicly available.

Code availability Statistical codes that are used for this study are available from the authors upon reasonable request and with permission of the Dutch Institute for Clinical Auditing and the Dutch ColoRectal Audit Board.

\section{Declarations}

Ethics approval and consent to participate The National Audit from the healthcare inspector required no informed consent of patients to collect data. Data analyses were performed on a pseudonymized dataset and did not need ethical approval according to Dutch law.

Conflict of interest The authors declare no competing interests.

Open Access This article is licensed under a Creative Commons Attribution 4.0 International License, which permits use, sharing, adaptation, distribution and reproduction in any medium or format, as long as you give appropriate credit to the original author(s) and the source, provide a link to the Creative Commons licence, and indicate if changes were made. The images or other third party material in this article are included in the article's Creative Commons licence, unless indicated otherwise in a credit line to the material. If material is not included in the article's Creative Commons licence and your intended use is not permitted by statutory regulation or exceeds the permitted use, you will need to obtain permission directly from the copyright holder. To view a copy of this licence, visit http://creativecommons.org/licenses/by/4.0/.

\section{References}

1. Kube R, Mroczkowski P, Granowski D et al (2010) Anastomotic leakage after colon cancer surgery: a predictor of significant morbidity and hospital mortality, and diminished tumour-free survival. Eur J Surg Oncol 36:120-124. https://doi.org/10.1016/j.ejso.2009.08.011

2. Bakker IS, Grossmann I, Henneman D et al (2014) Risk factors for anastomotic leakage and leak-related mortality after colonic cancer surgery in a nationwide audit. Br J Surg 101:424-432. https://doi.org/10.1002/bjs.9395

3. Frasson M, Granero-Castro P, Ramos Rodríguez JL et al (2016) Risk factors for anastomotic leak and postoperative morbidity and mortality after elective right colectomy for cancer: results from a prospective, multicentric study of 1102 patients. Int J Colorectal Dis. https://doi.org/10.1007/s00384-015-2376-6

4. Frasson M, Flor-Lorente B, Rodríguez JLR et al (2015) Risk factors for anastomotic leak after colon resection for cancer: multivariate analysis and nomogram from a multicentric, prospective, national study with 3193 patients. Ann Surg 262:321-330. https:// doi.org/10.1097/SLA.0000000000000973

5. Voron T, Bruzzi M, Ragot E et al (2019) Anastomotic location predicts anastomotic leakage after elective colonic resection for cancer. J Gastrointest Surg 23:339-347. https://doi.org/10.1007/s11605-018-3891-x

6. Midura EF, Hanseman D, Davis BR et al (2015) Risk factors and consequences of anastomotic leak after colectomy: a national analysis. Dis Colon Rectum 58:333-338. https://doi.org/10.1097/ DCR.0000000000000249

7. Hyman N, Manchester TL, Osler T et al (2007) Anastomotic leaks after intestinal anastomosis: It's later than you think. Ann Surg 245:254-258. https://doi.org/10.1097/01.sla.0000225083.27182.85

8. Sparreboom CL, Van Groningen JT, Lingsma HF et al (2018) Different risk factors for early and late colorectal anastomotic leakage in a nationwide audit. Dis Colon Rectum 61:1258-1266. https:// doi.org/10.1097/DCR.0000000000001202

9. Li YW, Lian P, Huang B et al (2017) Very early colorectal anastomotic leakage within 5 post-operative days: a more severe subtype needs relaparatomy. Scientific Reports 7. https://doi.org/10.1038/srep39936

10. Gessler B, Eriksson O, Angenete E (2017) Diagnosis, treatment, and consequences of anastomotic leakage in colorectal surgery. Int J Colorectal Dis 32:549-556. https://doi.org/10.1007/s00384-016-2744-x

11. Zarzavadjian Le Bian A, Tabchouri N, Denet C et al (2020) Anastomotic leakage after laparoscopic colectomy: who will require emergency fecal diversion? J Laparoendosc Adv Surg Tech. https://doi.org/10.1089/lap.2020.0765

12. Buchs NC, Gervaz P, Secic M et al (2008) Incidence, consequences, and risk factors for anastomotic dehiscence after colorectal surgery: a prospective monocentric study. Int J Colorectal Dis 23:265-270. https://doi.org/10.1007/s00384-007-0399-3

13. Sciuto A, Merola G, De PGD et al (2018) Predictive factors for anastomotic leakage after laparoscopic colorectal surgery. World J Gastroenterol 24:2247-2260. https://doi.org/10.3748/wjg.v24.i21.2247

14. Borstlap WAA, Westerduin E, Aukema TS et al (2017) Anastomotic leakage and chronic presacral sinus formation after low anterior resection. Ann Surg 266:870-877. https://doi.org/10.1097/SLA. 0000000000002429

15. Alves A, Panis Y, Trancart D et al (2002) Factors associated with clinically significant anastomotic leakage after large bowel resection: multivariate analysis of 707 patients. World J Surg 26:499502. https://doi.org/10.1007/s00268-001-0256-4

16. Bokey EL, Chapuis PH, Fung C et al (1995) Postoperative morbidity and mortality following resection of the colon and rectum for cancer. Dis Colon Rectum 38:480-487. https://doi.org/10.1007/BF02148847

17. Veyrie N, Ata T, Muscari F et al (2007) Anastomotic leakage after elective right versus left colectomy for cancer: prevalence and 
independent risk factors. J Am Coll Surg 205:785-793. https:// doi.org/10.1016/j.jamcollsurg.2007.06.284

18. Van Leersum NJ, Snijders HS, Henneman D et al (2013) The Dutch surgical colorectal audit. Eur J Surg Oncol 39:1063-1070. https://doi.org/10.1016/j.ejso.2013.05.008

19. SONCOS (2020) Multidisciplinaire normering oncologische zorg in nederland. In: soncos normeringsrapport 8. https://www.soncos.org/ wp-content/uploads/2020/03/SONCOS-normeringsrapport-versie8-1.pdf. Accessed 3 Jul 2021

20. Segelman J, Mattsson I, Jung B et al (2018) Risk factors for anastomotic leakage following ileosigmoid or ileorectal anastomosis. Colorectal Dis 20:304-311. https://doi.org/10.1111/codi.13938

21. Lauka L, Reitano E, Carra MC et al (2019) Role of the intestinal microbiome in colorectal cancer surgery outcomes. World Journal of Surgical Oncology 17:204. https://doi.org/10.1186/s12957-019-1754-x

22. Gershuni VM, Friedman ES (2019) The microbiome-host interaction as a potential driver of anastomotic leak. Curr Gastroenterol Rep 21:4. https://doi.org/10.1007/s11894-019-0668-7

23. Gallo G, Pata F, Vennix S et al (2020) Predictors for anastomotic leak, postoperative complications, and mortality after right colectomy for cancer: results from an international snapshot audit. Dis Colon Rectum 63:606-618. https://doi.org/10.1097/DCR.0000000000001590

24. Eto K, Urashima M, Kosuge M et al (2018) Standardization of surgical procedures to reduce risk of anastomotic leakage, reoperation, and surgical site infection in colorectal cancer surgery: a retrospective cohort study of 1189 patients. Int J Colorectal Dis 33:755-762. https://doi.org/10.1007/s00384-018-3037-3
25. Kryzauskas M, Bausys A, Degutyte AE et al (2020) Risk factors for anastomotic leakage and its impact on long-term survival in left-sided colorectal cancer surgery. World Journal of Surgical Oncology 18. https://doi.org/10.1186/s12957-020-01968-8

26. Agbamu DA, Day N, Walsh CJ et al (2012) Feasibility of preoperative chemotherapy for locally advanced, operable colon cancer: the pilot phase of a randomised controlled trial. Lancet Oncol 13:1152-1160. https://doi.org/10.1016/S1470-2045(12)70348-0

27. Krarup PM, Jorgensen LN, Harling H (2014) Management of anastomotic leakage in a nationwide cohort of colonic cancer patients. J Am Coll Surg 218:940-949. https://doi.org/10.1016/j. jamcollsurg.2014.01.051

28. Bakx R, Busch ORC, Bemelman WA et al (2004) Morbidity of temporary loop ileostomies. Dig Surg 21:277-281. https://doi.org/10. $1159 / 000080201$

29. Ihnát $\mathrm{P}$, Guňková $\mathrm{P}$, Peteja $\mathrm{M}$ et al (2016) Diverting ileostomy in laparoscopic rectal cancer surgery: high price of protection. Surg Endosc 30:4809-4816. https://doi.org/10.1007/s00464-016-4811-3

30. Näsvall P, Dahlstrand U, Löwenmark T et al (2017) Quality of life in patients with a permanent stoma after rectal cancer surgery. Qual Life Res 26:55-64. https://doi.org/10.1007/s11136-016-1367-6

31. Stewart K, Bray B, Buckingham R (2016) Improving quality of care through national clinical audit. Future Hospital Journal 3:203-206. https://doi.org/10.7861/futurehosp.3-3-203

Publisher's Note Springer Nature remains neutral with regard to jurisdictional claims in published maps and institutional affiliations. 